\title{
Visualization of vortex flow and shear stress in the aortic root during left ventricular assist device support
}

Shohei Yoshida, MD, Satsuki Fukushima, MD, PhD, Shigeru Miyagawa, MD, PhD,

Koichi Toda, MD, PhD, and Yoshiki Sawa, MD, PhD

From the Department of Cardiovascular Surgery, Osaka University Graduate School of Medicine, Osaka, Japan. Disclosures: Authors have nothing to disclose with regard to commercial support.

Received for publication Oct 27, 2016; revisions received Jan 3, 2017; accepted for publication Jan 18, 2017; available ahead of print July 1, 2017

Address for reprints: Yoshiki Sawa, MD, PhD, 2-2 Yamadaoka, Suita, Osaka 565-0871, Japan (E-mail: sawa-p@ surg1.med.osaka-u.ac.jp).

J Thorac Cardiovasc Surg 2017;154:877-8

$0022-5223 / \$ 36.00$

Copyright (C) 2017 by The American Association for Thoracic Surgery

http://dx.doi.org/10.1016/j.jtcvs.2017.01.060

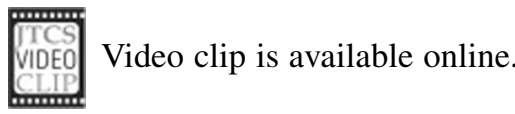

$\mathcal{B}$ Supplemental material is available online.

Progression of aortic valve insufficiency significantly compromises hemodynamics in patients with advanced cardiac failure receiving left ventricular assist device (LVAD)

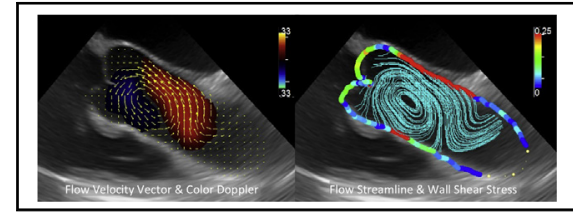

Retrograde blood flow from the LVAD Increases the wall shear stress on aortic valve leaflets.

\section{Central Message}

We visualized the retrograde blood flow in the ascending aorta in the patient under continuous-flow left ventricular assist device support, which developed into a vortex producing shear stress on the aortic valve leaflets.

See Editorial Commentary page 879.

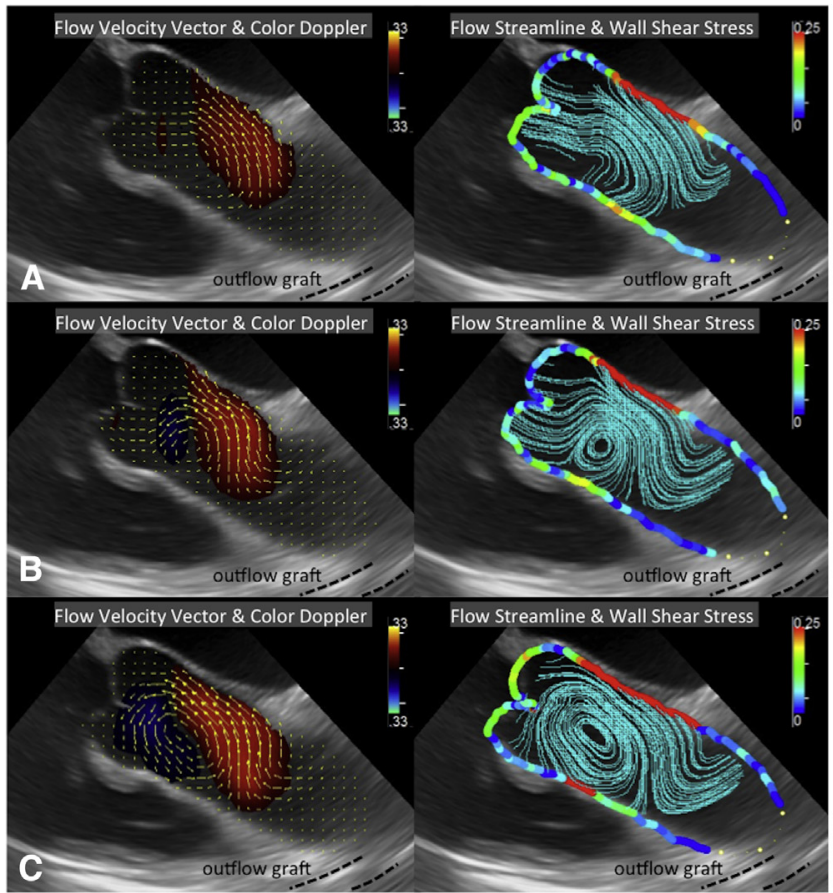

support. ${ }^{1}$ Nonphysiologic shear stress in the aortic valve produced by collision of the continuous retrograde blood flow from the LVAD has been suggested to induce

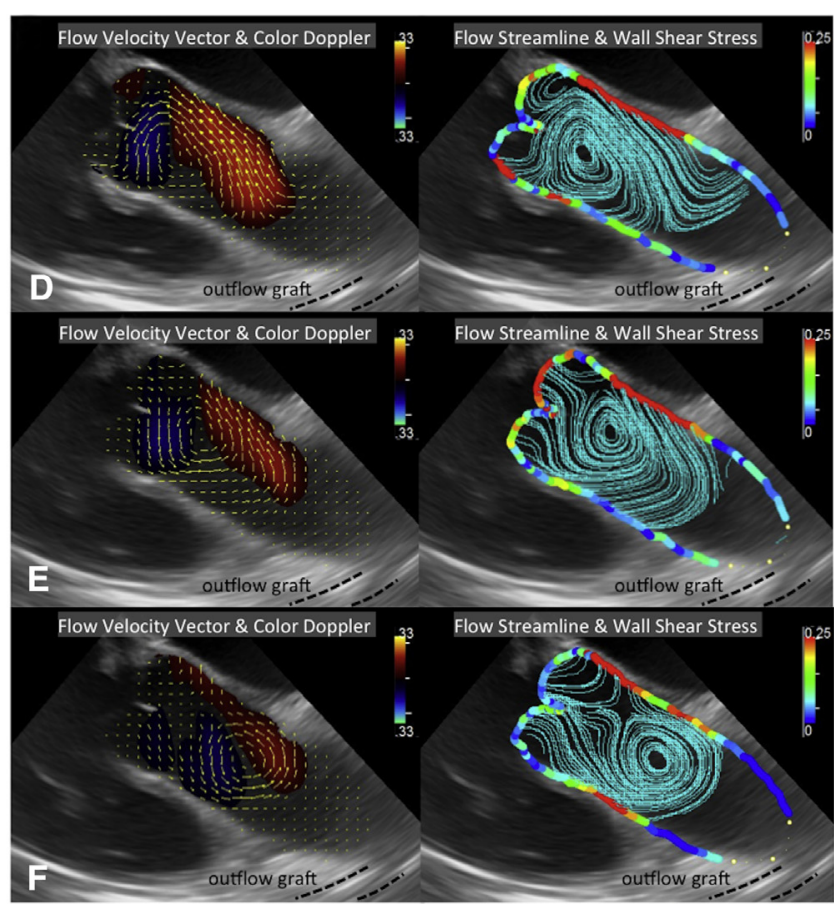

FIGURE 1. (Left) Flow velocity vector on color Doppler. Red represents flow toward the top; blue, flow toward the bottom. The yellow arrow represents the flow velocity vector, with the direction of the arrow equal to the flow direction and the size of arrow reflecting flow velocity. (Right) The fine green lines indicate the flow streamline. Multiple colors around the aortic root represent the wall shear stress: red, high; blue, low. A, Systolic phase. B-E, Early and middiastolic phases. F, Late diastolic phase. 


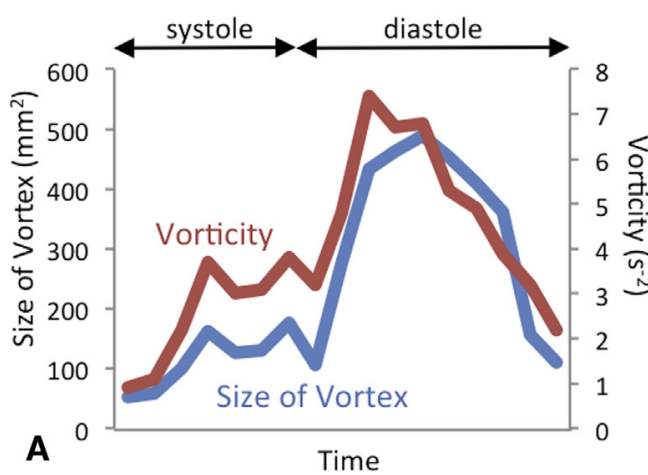

$(\mathrm{Pa})$

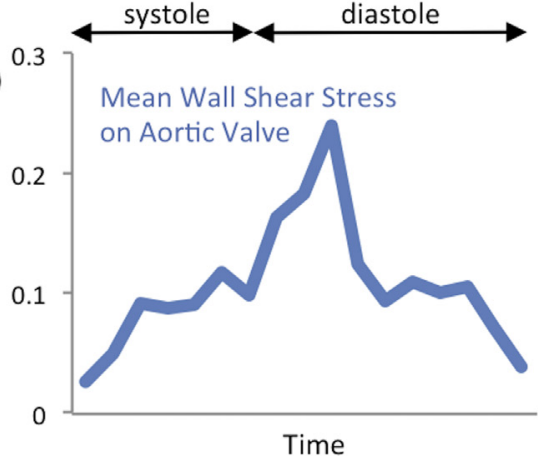

FIGURE 2. A, Graph representing the time-dependent changes during a cardiac cycle in the size (blue line) and vorticity (red line) of the vortex in the ascending aorta in patients with ischemic cardiomyopathy on LVAD support. Both variables approached the peak just after the systolic phase and settled in the late diastolic phase. B, Graph representing the time-dependent changes in mean wall shear stress on the aortic valve leaflets during a cardiac cycle in patients with ischemic cardiomyopathy on LVAD support. The variable approached the peak just after the systolic phase and settled in the late diastolic phase.

valve leaflet thinning and, consequently, aortic valve insufficiency and its progression, ${ }^{2}$ although computational fluid dynamics to visualize blood flow in the aortic root has not been established because of its complex anatomy, such as the origin of the coronary arteries. ${ }^{3,4}$ We clearly visualized the blood flow in the aortic root in patients under LVAD support by conducting an echocardiographic study with vector flow mapping analysis (DAS-RS1).

Here we present images of a case of ischemic dilated cardiomyopathy in a 40-year-old woman just after implantation of a continuous-flow LVAD (HeartMate II; Thoratec, Pleasanton, Calif) (Figure E1). The blood flow in the ascending aorta showed pulsatility from the beating of the

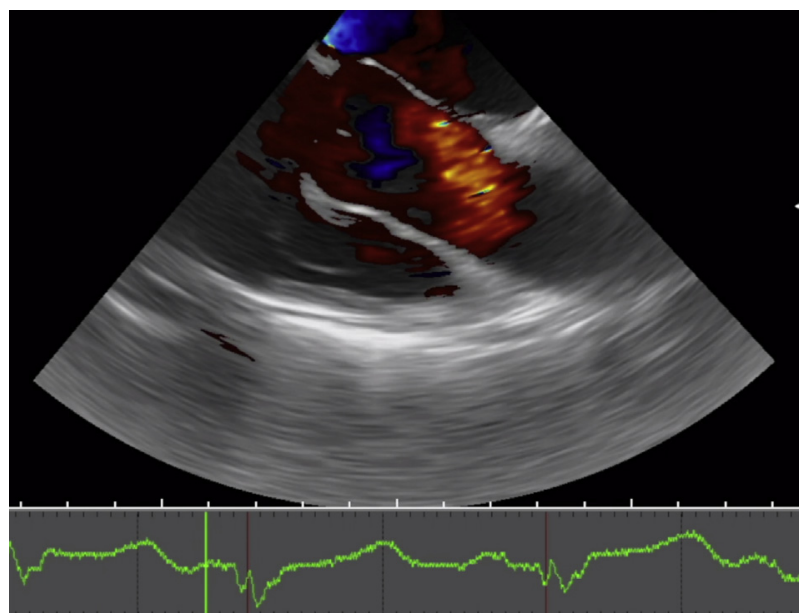

VIDEO 1. Echocardiography and vector flow mapping analysis in the aortic root during 3 cardiac cycles. First, color Doppler echo. Second, color Doppler and flow velocity vector analysis. Third, wall shear stress and streamline analysis. Video available at: http://www.jtcvsonline.org/ article/S0022-5223(17)31091-7/addons. left ventricle, which increased blood flow via the LVAD without opening the aortic valve. During systole, retrograde blood flow was clearly visualized in the ascending aorta, and shear stress increased in the posterior wall of the ascending aorta (Figure 1, A). Blood flow then developed into a vortex in the aortic root, moving toward the aortic valve with magnified vorticity, consequently producing shear stress on the aortic valve leaflets (Figure 1, $B$-E). In the late diastolic phase, the vortex moved distally with its vorticity settled (Figure 1, F; Figure 2, $A$ and $B$; and Video 1).

Blood flow through the aortic valve may alter the vortex size and/or vorticity in the aortic root, potentially decreasing the shear stress in the aortic valve. ${ }^{1,5}$ Further studies are needed to evaluate case-dependent differences in blood flow to demonstrate the mechanisms underlying the initiation and progression of aortic valve insufficiency after LVAD implantation.

\section{References}

1. Toda K, Fujita T, Domae K, Shimahara Y, Kobayashi J, Nakatani T. Late aortic insufficiency related to poor prognosis during left ventricular assist device support. Ann Thorac Surg. 2011;92:929-34.

2. Hata H, Fujita T, Ishibashi-Ueda H, Nakatani T, Kobayashi J. Pathological analysis of the aortic valve after long-term left ventricular assist device support. Eur J Cardiothorac Surg. 2014;46:193-7.

3. Karmonik C, Partovi S, Loebe M, Schmack B, Ghodsizad A, Robbin MR, et al. Influence of LVAD cannula outflow tract location on hemodynamics in the ascending aorta: a patient-specific computational fluid dynamics approach. ASAIO J. 2012; 58:562-7.

4. Callington A, Long Q, Mohite P, Simon A, Mittal TK. Computational fluid dynamics study of hemodynamic effects on aortic root blood flow of systematically varied left ventricular assist device graft anastomosis design. J Thorac Cardiovasc Surg. 2015;150:696-704.

5. Patil NP, Sabashnikov A, Mohite PN, Garcia D, Weymann A, Zych B, et al. De novo aortic regurgitation after continuous-flow left ventricular assist device implantation. Ann Thorac Surg. 2014;98:850-7. 

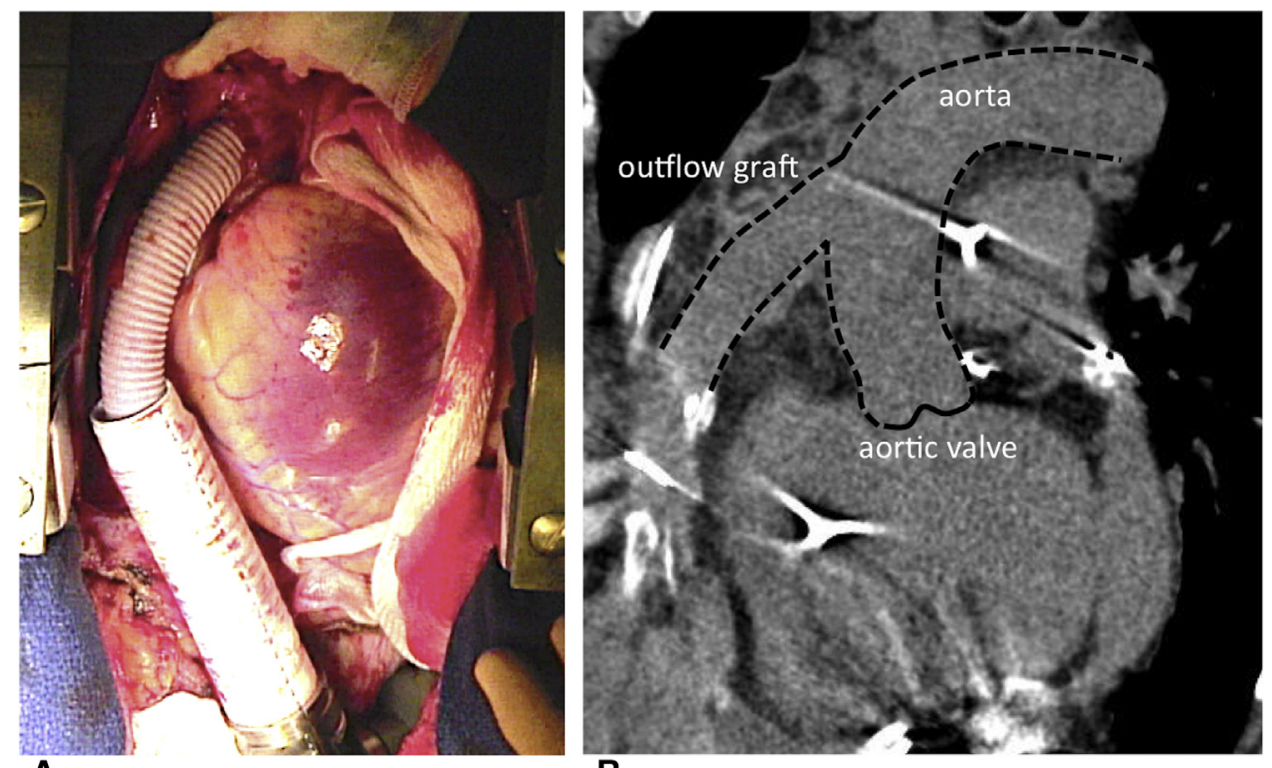

A

B

FIGURE E1. A, LVAD outflow graft anastomosed to the anterior wall of the ascending aorta. B, Postoperative anatomy, including the outflow graft, the ascending aorta, and the aortic valve. 